\title{
Evidências de validade e precisão da Escala de Coping através de Ouvir Música
}

\author{
Carlos EduardoPimente - UniveridadedeBraślia, Braślia, Brasil \\ MarlizteMaldonadoVargas - UniversidadeTiradentes, Segipe, Brasil \\ Thiago Olivera deAlmida - UnivesidadeTiradentes, Sergipe, Brasil \\ VivianeAndradePradoMaynart- UniveridadeTiradentes, Seg̣pe, Brasil \\ SarahMonteirodeC. T. Figuireeb- UniversidadeTiradentes, Sergipe, Brasil
}

\begin{abstract}
Resumo
O presente estudo buscou verificar a validade de construto e precisão da Escala de Coping através de Ouvir Música (ECOM), cujos fatores representam estratégias de coping focadas na emoção, no problema e evitação/ desligamento. Contou com a participação de 248 estudantes de escolas e universidades públicas e particulares da cidade de Aracaju, no Nordeste brasileiro. Os principais resultados corroboram a estrutura original da escala com três fatores. Foram verificados índices de precisão adequados para o fator copingfocado na emoção, mas índices menores para os fatores de copingfocado no problema e evitação. Foram verificadas ainda diferenças por gênero nas estratégias de coping relacionadas a ouvir música. Os dados são discutidos considerando a literatura sobre psicometria e sugestões de pesquisa são realizadas para se desenvolverem os estudos na área de coping
\end{abstract}

Palavasdhave Copingatravés de ouvir música; Validade; Precisão.

\section{Evidence of validity and accuracy of the Coping by Music Listening Scale}

\begin{abstract}
This study aims at evaluating the construct validity and accuracy of the Coping by Music Listening Scale (CMLS) whose factors represent coping strategies as emotion in the problem and avoidance/ disconnexion. It acounted on the participation of 248 students from schools and public and private universities in the city of Aracaju, Brazilian Northeast. The main results confirm the scale's original structure with three factors. Appropriate accuracy indices of emotionoriented coping factor and the scale as a whole were verified, but lower rates for the problem-oriented and avoidance factors. It was also found gender differences in coping strategies by music listening. The results are discussed considering the psychometric literature and suggestions for research were carried out to develop studies on the coping research.

Keguards Coping by music listening; Validity; Reliability.
\end{abstract}

\section{Evidencias de validez y precisión de la "E scala de Coping através de ouvir música"}

\begin{abstract}
Resumen
El presente estudio buscó verificar la validez de constructo y precisión de la "Escala de Copingatravés de Ouvir Música" (ECOM), cuyos factores representan estrategias de coping focalizadas en la emoción, en el problema y evitación/ desligamiento. Contó con la participación de 248 estudiantes de escuelas y universidades públicas y particulares de la ciudad de Aracaju, en el Nordeste brasileño. Los principales resultados corroboran la estructura original de la escala con tres factores. Fueron verificados índices de precisión adecuados para el factor copingfocalizado en la emoción y de la escala total, pero índices menores para los factores de copingfocalizado en el problema y evitación. Fueron verificadas aún diferencias por género en las estrategias de copingrelacionadas a oír música. Los datos son discutidos considerando la literatura sobre psicometría y sugestiones de investigaciones son realizadas para el desarrollo de estudios en el área de coping

Palabrasdave Copinga través de oír música; Validez; Precisión.
\end{abstract}

A observação do cotidiano, assim como resultados de pesquisas, têm demonstrado a importância da música na vida das pessoas (Mitchell, Mcdonald, Knussen \& Serpell, 2007; North, Hargreaves \& O’Neill, 2000; Rentfrow \& Gosling, 2003). A música tem sido entendida como uma maneira de comunicar-se, expressar atitudes e valores, bem como a própria personalidade (Boer, 2009; Cattel \& Saunders, 1954; Pimentel \& Donnely, 2008; Rentfrow \& Gosling, 2003).

Existem pesquisas que demonstraram benefícios de ouvir música para as habilidades cognitivas (Schellenberg \& Hallam, 2005), alívio da dor crônica, qualidade de vida (Mitchell \& cols., 2007), raciocínio espaço-temporal (Hetland, 2000), divertimento, criatividade, expressão de emoções e redução da solidão (Bueno \& Macedo, 2004; North \& cols., 2000; Schwartz \& Fouts, 2003). Além disso, ouvir música impacta o estado de ânimo (Bueno \& Bergamasco, 2008), regula o humor (Premuzic-Chamorro \& Furnham, 2007) e propicia interações sociais (Boer, 2009). Evidências empíricas indicam que ouvir música é uma das mais importantes estratégias de enfrentamento dos problemas (coping para os adolescentes. No survey desenvolvido por Mitchell e colaboradores (2007), por exemplo, verificou-se que a música foi utilizada pelos participantes do seu estudo com objetivos de se lidar com as situações do cotidiano, depressão, ansiedade, para relaxar e se animar. 
De acordo com Miranda e Claes (2009), esse dado encontra respaldo na teoria dos usos e gratificações, segundo a qual os adolescentes escolhem as mídias de acordo com as situações sociais (eventos estressantes) e características pessoais. D estacaram também que outra teoria relacionada é a do gerenciamento do humor, a qual prediz que os adolescentes, na tentativa de regular as emoções, utilizam ativamente a música. Em suma, os autores enfatizaram o comportamento de se ouvir música como uma estratégia de copingrelevante para os adolescentes lidarem com situações estressantes, seja com a família, seja com escola ou amigos.

Miranda e Claes (2009), realizando análises de regressão múltipla por sexo, verificaram no grupo de moças que 0 coping através de ouvir música tipo evitação predisse altos níveis de depressão $(\beta=0,34$, $\mathrm{t}=4,84 ; \mathrm{p}<0,001)$. Por outro lado, constataram que 0 coping através de ouvir música focado no problema predisse baixos níveis de depressão $(\beta=-0,14, \mathrm{t}=-1,99$; $\mathrm{p}<0,05)$. Ademais, perceberam, no grupo de rapazes, que 0 copingatravés de ouvir música focado na emoção predisse altos níveis de depressão $(\beta=0,20, t=2,89$; $\mathrm{p}<0,01)$. Por outro lado, verificaram que o coping através de ouvir música focado no problema e o tipo evitação não predisseram a depressão.

\section{Coping}

O coping pode ser entendido como: "esforços cognitivos e comportamentais dinâmicos para gerir demandas específicas externas e/ ou internas, as quais são avaliadas como excedendo os recursos pessoais" (Lazarus \& Folkman, 1984, p. 141). Esses autores caracterizaram o coping como processo que envolve avaliação da situação estressante e não inclui comportamentos do tipo automáticos, e pensamentos que não envolvem esforço. Acrescentam, ainda, que se pode abranger um espectro de comportamentos e pensamentos independentes de terem um bom resultado na administração das situações estressantes. Gerir demandas pode incluir a redução, evitação, tolerância ou mesmo a aceitação de condições estressantes, assim como tentativas conscientes de se dominar o ambiente (Lazarus \& Folkman, 1984).

Mais sinteticamente 0 coping pode ser definido como um "conjunto das estratégias utilizadas pelas pessoas para adaptarem-se a circunstâncias adversas" (Antoniazzi, Dell'aglio \& Bandeira, 1998, p. 274) e varia de acordo com o tipo de situação (Terry, 1991). Esses autores salientaram, ainda, que o conceito de copingvem sendo objeto de estudo na psicologia social, da personalidade ou clínica (Antoniazzi \& cols., 1998). Certamente, 0 coping é um dos construtos mais importantes da psicologia e já gerou um grande número de pesquisas na realidade brasileira (Antoniazzi
\& cols., 1998; Antoniazzi, Souza \& Hutz, 2009; Balbinotti, Barbosa, Wiethaeuper \& Teodoro, 2006; Dela Coleta \& Dela Coleta, 2008; Diniz \& Zanini, 2010; Lisboa \& cols., 2002; Panzini \& Bandeira, 2007; Raimundo \& Pinto, 2006; Santos \& Martendal, 2008; Tamayo \& Tróccoli, 2002; Vera-Noriega, Albuquerque, Laborin \& Pimentel, 2003).

O modelo de Lazarus e Folkman (1984) se constitui em uma das contribuições mais importantes ao estudo de coping (Antoniazzi \& cols., 1998) e também é necessário para 0 entendimento do presente estudo (Miranda \& Claes, 2009). Tal modelo trata de dois tipos principais de coping estratégias de coping focado na emoção diz respeito a tentativas de diminuir as emoções negativas geradas por estressores; 0 coping focado no problema se refere aos esforços para tentar mudar a situação originária do estressor. Estratégias de caping incluem comportamentos e pensamentos que 0 indivíduo usa para lidar com o estressor (Folkman, Lazarus, Dunkel-Schetter, DeLongis \& Gruen, 1986). 0 uso do copingfocado no problema tem se relacionado mais com a autoestima (Aspinwall \& Taylor, 1992; Barker, 2007; Terry, 1991). Baseados nesses modelos podem-se verificar estratégias de ação instrumental (como se preparar para um exame), busca de suporte emocional (como aceitar um conselho de alguém), fuga e auto-culpabilização (criticar-se por não ter trabalhado o suficiente) e minimização (busca minimizar a ameaça do problema, Terry, 1991).

O utro tipo de copingfrequentemente pesquisado e também necessário para esta pesquisa diz respeito ao copingtipo evitação, que envolve a negação ou evitação do estressor, oriundo da tipologia de evitação vs aproximação (ver também Antoniazzi \& cols., 1998; Carver \& Connor-Smith, 2010). 0 menor uso desse tipo evitação tem se relacionado com a autoestima (Aspinwall \& Taylor, 1992). Em Lazarus e Folkman (1984) verifica-se que o tipo evitação é considerado como um tipo de copingfocado na emoção.

Em suma, Antoniazzi e colaboradores (1998) destacaram que estas estratégias de copingvariam no que diz respeito a sua eficácia em função dos estressores na situação. Ademais, as autoras destacaram que recentemente surgiu um novo tipo de estratégia, focalizada nas relações interpessoais. A despeito de poderem ser verificadas outras estratégias de coping até baseadas na tipologia de Lazanus e Folkman (1984), considerada a mais compreensível na área (Antoniazzi \& Cols., 1998), o presente estudo baseia-se nas estratégias de copingfocado na emoção, no problema e no tipo evitação, uma vez que este modelo foi usado na elaboração da Copingby MusicListening Scale(Miranda \& Claes, 2009), foco principal deste artigo, como se verá na continuação. 


\section{Escala deCopingatravés deOuir Música}

Recentemente Miranda e Claes (2009) utilizaram estes conceitos (copingfocado na emoção, no problema e tipo evitação) para desenvolver sua medida de coping através da música, intitulada Coping by Music Listening Scale e traduzida como Escala deCoping através de Ouir Música (ECOM), para mensurar situações nas quais se escuta música para melhor lidar com os problemas. Essa medida, portanto, partiu do conceito de coping multidimensional e foi composta por 10 itens, respondidos em uma escala de frequência de 5 pontos, distribuídos nos três fatores de coping Copingfocado na Emoção, Copingfocado no Problema e Evitação.

Miranda e Claes (2009) realizaram uma pesquisa com 467 estudantes adolescentes no Canadá com a ECOM, que se apresentou válida e razoavelmente precisa segundo critérios estabelecidos na literatura (Anastasi \& Urbina, 2000; Nunnally, 1970, Pasquali, 2003) e respondendo por $62,71 \%$ da variância explicada por meio da análise fatorial dos eixos principais (PAF). E com índices de precisão avaliados pela técnica alfa de Cronbach $(\alpha)$ de 0,88 para o fator de coping focado na emoção (que compreende escutar música quando estressado, com problemas, para reduzir o estresse, a depressão, ansiedade ou medo, relaxar e ter emoções positivas), 0,85 para o fator de coping tipo esquiva ou evitação (que compreende escutar música quando estressado, com problemas, para evitar pensar nos problemas ou em pessoas que estão causando problemas) e 0,69 para o copingfocado no problema (que compreende escutar música quando estressado com problemas para ajudar a trabalhar melhor, a refletir). Os autores ainda destacaram que este último fator obteve um eigenvalue considerado limítrofe $(0,92)$, mas decidiu-se mantê-lo certamente pela ponderação da estrutura fatorial estar condizente com a teoria do construto.

Portanto, essa medida tem propriedades psicométricas favoráveis para a sua validação no presente contexto e uma boa teoria subjacente que permitiu derivar itens claros. Outra vantagem diz respeito à praticidade da mesma; além de ser uma técnica tipo lápis e papel, de baixo custo e simples aplicação, trata-se de uma medida breve, formada por apenas 10 itens. Utilizando esta medida, Miranda e Claes (2009) conseguiram verificar ainda que, nas garotas, o copingatravés da música focado no problema se relacionou com menos depressão, enquanto o coping através da música tipo evitação se relacionou com mais depressão. Nos garotos, o coping através da música focado na emoção se relacionou com mais altos níveis de depressão. Considerando uma análise de variância múltipla (MANOVA), os autores ainda verificaram que as adolescentes do sexo feminino obtiveram as médias mais altas (coping focado na emoção: $M=3,64$, $\mathrm{DP}=0,99$; coping focado no problema: $\mathrm{M}=3,47$, $\mathrm{DP}=0,92$; e evitação: $\mathrm{M}=3,38, \mathrm{DP}=1,07$ ) do que os do sexo masculino nos três fatores de coping (coping focado na emoção: $M=3,18, \mathrm{DP}=1,07$; copingfocado no problema: $\mathrm{M}=3,24, \mathrm{DP}=1,00 ; \quad \mathrm{e}$ evitação: $\mathrm{M}=2,82, \mathrm{DP}=1,20$ ) dando-se as menores diferenças no coping focado no problema. Com relação à população total, foram verificadas as seguintes pontuações: coping focado na emoção: $\mathbf{M}=3,41$, $\mathrm{DP}=1,05$; coping focado no problema: $\mathrm{M}=3,36$, $\mathrm{DP}=0,96$ e evitação: $\mathrm{M}=3,11, \mathrm{DP}=1,17$. Portanto, justificou-se o que era teoricamente esperado no sentido de mais importância do coping focado na emoção para o copingatravés de se ouvir música.

O objetivo principal deste estudo consiste em se proceder à validade de construto e verificação da precisão da ECOM em uma amostra de estudantes do ensino médio e universitário. Este critério foi definido com o objetivo de se testar a escala em apreço numa amostra de adolescentes, como feito pelos autores da escala (Miranda \& Claes, 2009). No entanto, pretendese ampliar esta amostra na medida em que se incluem estudantes universitários que podem ser considerados jovens. Além desse objetivo, busca-se verificar se existem relações entre os tipos de copingcom o sexo e a idade dos participantes. De acordo com a teoria de coping revista para este estudo, 0 coping tem sido definido como processo que muda da infância até a terceira idade (Lazarus \& Folkman, 1984). Tem sido verificado também que a idade é uma variável que modera as relações entre copinge personalidade (Carver \& Connor-Smith, 2010). Além disso, a ECOM foi originalmente validada e desenvolvida para adolescentes e foram verificadas diferenças entre adolescentes do sexo masculino e feminino. Mesmo que no Brasil possam ser encontrados vários estudos específicos sobre coping a ECOM ainda não foi validada neste contexto, o que o torna ainda mais necessário.

\section{Partiapantes}

\section{Método}

Trata-se de uma amostra por conveniência ou não-probabilística. Os participantes foram 248 estudantes de instituições públicas e particulares do ensino médio $(39,6 \%)$ e universitários $(60,5 \%)$, com média de idade de 19 anos ( $\mathrm{DP}=3,83$, com idades predominantemente de 14 a 30 anos [98\%]), divididos equitativamente entre homens e mulheres, principalmente solteiros (81\%) de diversos cursos, mas sobretudo de Engenharia Elétrica (15,8\%) e Psicologia $(12 \%)$. 


\section{Instrumento}

Escala de Coping através de Oumir Música (ECOM). Trata-se de uma medida que objetiva aferir as estratégias de coping através de ouvir música em situações que envolvem escola, amigos ou família. Foi desenvolvida por Miranda e Claes (2009) para se verificar as estratégias de coping através de se ouvir música, dos adolescentes especificamente. Inicialmente os autores contaram com 15 itens, mas cinco foram eliminados no processo de validação. A versão final consistiu de uma medida intervalar com 10 itens ancorados numa escala de cinco pontos, variando de $1=$ Nunca a 5 = Sempree distribuídos em três fatores que representam estratégias de coping Copingfocado na emoção, coping focado no problema e evitação/ desligamento. Exemplos de itens por cada fator são: Quando estou estressado, com problemas (na escola, com amigos ou com a família), escuto meu tipo de música favorito para...1)...evitar pensar nos meus problemas (Evitação/ D esligamento), 3)...ajudarme a refletir melhor para encontrar soluções para os meus problemas (Coping focado no Problema) e 6)...ajudar-me a reduzir minhas emoções negativas, tais como sentimentos de depressão, ansiedade e medo (Coping focado na Emoção), mais um questionário sociodemográfico (com perguntas sobre sexo, idade, curso) e 0 Termo de Consentimento Livre e Esclarecido. A escala foi traduzida do inglês para 0 português por pesquisadores bilíngues. Essa versão em português foi testada (validação semântica), consequentemente, em uma amostra de estudantes do ensino médio e universitários com 0 fim de se obter clareza e correção na linguagem.

\section{Procedimento}

Buscou-se a participação voluntária de alunos de ensino médio e universitário de ambos os sexos. Para tanto, o passo inicial foi contatar a direção de escolas de ensino médio e universidades com o fim de agendar horários para a coleta de dados. Alunos de psicologia, colaboradores desta pesquisa, cujo projeto foi aprovado pelo Comitê de Ética em Pesquisa da Universidade Tiradentes, administraram questionários nas salas de aula e praças das universidades. Foi utilizado um procedimento padrão para coleta de dados na pesquisa social. Todos os pressupostos éticos, de acordo com as diretrizes e normas regulamentadas de pesquisa envolvendo seres humanos, conforme Resolução no 196, de 10 de outubro de 1996, do conselho Nacional de Saúde do Ministério de Saúde Brasília - DF foram levados em consideração. Isto é, na administração dos questionários, os respondentes foram informados oralmente pelos alunos de psicologia responsáveis pela coleta que inicialmente responderam o 'l'ermo de Consentimento Livre e Informado, garantindo 0 direito à privacidade, que todas as informações são anônimas, que serão apenas tratadas estatisticamente e não haverá nenhum constrangimento para 0 respondente, nem obrigatoriedade em responder ao questionário, mas que sua participação era importante para 0 desenvolvimento da pesquisa psicológica no país. Além disso, foi destacado que a qualquer momento o participante poderia declinar em continuar a responder o questionário. No final da coleta de dados os colaboradores da pesquisa agradeceram aos alunos pela participação voluntária, assim como aos professores pelo espaço na aula e à direção da escola ou coordenação do curso universitário. Os participantes responderam aos questionários no ambiente coletivo de sala de aula e espaços abertos, como praças, mas 0 fizeram individualmente. Mesmo que o instrumento fosse do tipo autoadministrável, os aplicadores explicavam como os respondentes deveriam completar os questionários.

\section{Análises etatísticas}

A versão 15 do pacote estatístico SPSS para Windows $^{\circledR}$ (Statistical Padkage for the Social Saienes) foi utilizada para digitação e análise dos dados. Essas análises consistiram em análises descritivas (média, desvio padrão, percentuais), análise fatorial comum e confirmatória, alfa de Cronbach, lambda 2 de Guttman, MANCOVA e análises de correlações de Pearson.

0 AMOS (Analysis of MOment Structures) 7 foi empregado para testar o modelo original da ECOM, assim como modelos alternativos, como recomendado na literatura especializada (DiStefano \& Hess, 2005; Thompson, 2005; Weston \& Gore, 2006). Foram considerados os seguintes indicadores de ajuste (Browne \& Cudeck, 1993; Byrne, 2001, 2010; Hu \& Bentler, 1999; Tabachnick \& Fidell, 2007):

- Razão $\chi^{2}$ / gl. O quiquadrado do modelo dividido por seus graus de liberdade tem sido considerado a primeira tentativa de se lidar com o problema da influência do tamanho da amostra no quiquadrado (Byrne, 2010). Diversos autores têm sugerido índices distintos para 0 quiquadrado relativo $\left(\chi^{2} / \mathrm{gl}\right)$, recomendando-se, para um ajuste adequado, valores variando entre 1 e 5 (ver comentários de Garson, 2010);

- GFI e CFI. Os Goodnssof-Fit Index (GFI) e ComparativeFit Index (CFI) devem se situar próximos de 1, acima de 0,90, mas atualmente tem-se sugerido valores mais restritivos para o CFI $(>0,95$, Byrne, 2001, 2010; Hu \& Bentler, 1999; Thompson, 2005) para se considerar um modelo ajustado; 
- RMSEA. Este índice, Rot-Mean-Square Error of Approximation deve ser mais próximo de zero para considerar um adequado ajuste do modelo aos dados; valores próximos a 0,06 ou menores revelam ajuste aceitável (Mcdonald \& Ho, 2002), admitindo-se valores de até 0,10 (Browne \& Cudeck, 1993; Hu \& Bentler, 1999);

- ECVI, CAIC e teste de diferenca entre qui-quadradbs $\left(\Delta \chi^{2}\right)$. 0 Expeted CrossValidation Index (ECVI) e Consistent Akaike Infomation Criterion (CAIC) são utilizados para comparar modelos; o modelo com menores valores de ECVI e CAIC é tido como 0 melhor (Byrne, 2010; DiStefano \& Hess, 2005; Hu \& Bentler, 1995; MacCallum \& Austin, 2000; Tabachnick \& Fidell, 2007). $0 \Delta \chi^{2}$ significativo indica que 0 modelo com menor valor de $\chi^{2}$ tem melhor ajuste aos dados.

\section{Resultados}

Inicialmente foram verificadas estatísticas descritivas do conjunto de itens da ECO M. Uma vez que os valores missingestiveram abaixo de $5 \%$, optou-se por substituí-los pela média (Hair, Black, Babin, Anderson \& Tatham, 2009). Foi verificado, também, um percentual ínfimo de valores auties $(<1 \%)$ que não impactaram os resultados e por isso foram mantidos.

\section{Análisefatcrial ealfa deCronbach}

Antes de se levar a cabo a análise fatorial pelo método dos eixos principais (PAF), verificou-se 0 índice KMO $(0,87)$ e Teste de Esfericidade de Bartlett $\left[\chi^{2}(45)=724,420 ; \quad p<0,001\right]$, que apresentaram resultados favoráveis, para se seguir na análise. Inicialmente, 0 primeiro ensaio fatorial sem fixar 0 número de fatores com rotação dbiminapresentou dois fatores a se extrair com eigenalues iguais a 4 e 1,12. A análise do screp pld também sugeriu dois fatores, mas uma análise paralela (PA) com 1.000 simulações e 99\% de confiança gerou os seguintes eigenvalues aleatórios: $1,32,1,22,1,14,1,08$ e 1,02. A partir dos resultados da PA, que tem sido considerada uma técnica mais precisa para a decisão sobre o número de fatores (Hayton, Allen \& Scarpello, 2004; Zwick \& Velicer, 1986), indicou-se uma solução unifatorial. Porém, tendo em conta a solução original, decidiu-se realizar uma nova análise PAF, fixando-se em três fatores a se extrair. Os resultados dessa análise podem ser vistos na Tabela 1.

A estrutura formada por três fatores gerou cargas fatoriais consideradas satisfatórias. As comunalidades mostram 0 percentual de variância do item explicado pela solução, sendo os itens 1 e 5 os mais bem explicados (Hair \& cols., 2009). A despeito do terceiro fator ter apresentado um égenvalue abaixo de $1(0,94)$, decidiu-se por esta estrutura por sua clareza conceitual.

0 primeiro fator foi formado pelos itens concernentes ao fator coping focado na emoção, 0 segundo pelos itens do fator copingfocado no problema e 0 terceiro pelos itens do fator evitação/ desligamento, que explicaram conjuntamente 60,66\% da variância explicada. 0 primeiro fator apresentou um bom índice de precisão mediante a técnica alfa de Cronbach e lambda 2 de Guttman, mas os outros dois fatores apresentaram índices bem menores.

Por fim, foram calculadas as correlações entre os itens dos três fatores da ECOM. Verificou-se que 0 primeiro fator, coping focado na emoção, apresentou correlação positiva entre os itens variando de 0,46 a 0,60 ( $\left.r_{\text {média }}=0,52\right) ; 0$ segundo fator, coping focado no problema, apresentou correlação positiva entre os itens de 0,20 a 0,31 $\left(\mathrm{r}_{\text {média }}=0,25\right)$ e 0 terceiro fator, evitação, apresentou correlação positiva entre seus dois itens de 0,38 .

Tabela 1. Estrutura fatorial da ECOM

(continua)

\begin{tabular}{|c|c|c|c|c|}
\hline \multirow{2}{*}{ Itens } & \multicolumn{3}{|c|}{ Fatores } & \multirow[b]{2}{*}{$\mathrm{h}^{2}$} \\
\hline & 1 & 2 & 3 & \\
\hline 9)...ajudar-me a reduzir o estresse, a relaxar e me acalmar. & 0,83 & 0,03 & $-0,11$ & 0,44 \\
\hline $\begin{array}{l}\text { 6)...ajudar-me a reduzir minhas emoções negativas, tais como sentimentos de } \\
\text { depressão, ansiedade e medo. }\end{array}$ & 0,64 & $-0,06$ & 0,25 & 0,32 \\
\hline $\begin{array}{l}\text { 8)...ajudar-me a reduzir minhas emoções negativas, como frustração, raiva e } \\
\text { agressividade. }\end{array}$ & 0,63 & $-0,15$ & 0,29 & 0,34 \\
\hline 10)...ajudar-me a desopilar. & 0,62 & 0,21 & $-0,09$ & 0,19 \\
\hline 7)...ajudar-me a ter emoções positivas, como alegria, felicidade e esperança. & 0,57 & 0,12 & 0,05 & 0,27 \\
\hline 3)...ajudar-me a refletir melhor para encontrar soluções para os meus problemas & 0,04 & 0,58 & $-0,04$ & 0,61 \\
\hline 4)...ajudar-me a estudar (ou trabalhar) melhor. & 0,11 & 0,42 & 0,07 & 0,45 \\
\hline 5)...motivar-me a fazer o que acho que devo fazer. & $-0,03$ & 0,41 & 0,09 & 0,58 \\
\hline 1)...evitar pensar nos meus problemas. & 0,10 & 0,14 & 0,54 & 0,62 \\
\hline 2)...evitar pensar em pessoas que estão me causando problemas. & 0,02 & 0,07 & 0,53 & 0,50 \\
\hline
\end{tabular}


Tabela 1. Estrutura fatorial da ECOM

\begin{tabular}{|c|c|c|c|c|}
\hline \multirow[b]{2}{*}{ Itens } & \multicolumn{3}{|c|}{ Fatores } & \multirow[b]{2}{*}{$\mathrm{h}^{2}$} \\
\hline & 1 & 2 & 3 & \\
\hline № de itens & 5 & 3 & 2 & \\
\hline Valor próprio & 4,00 & 1,12 & 0,94 & \\
\hline \% de variância explicada & 40,02 & 11,25 & 9,40 & \\
\hline Alfa de Cronbach & 0,84 & 0,50 & 0,55 & \\
\hline Lambda 2 de Guttman & 0,85 & 0,51 & 0,55 & \\
\hline
\end{tabular}

\section{Análisefatcoial confimatónia}

Com o fim de se verificar em que medida a estrutura fatorial verificada na análise anterior se adapta aos dados, realizou-se uma análise fatorial confirmatória (CFA) com o estimador ML (Maximm Likeihood), analisando-se a matriz de covariâncias. Foram testados os seguintes modelos: a) unifatorial; b) bifatorial (formado pela junção do fator caing focado na emoção com o fator tipo evitação); c) original (trifatorial) e d) original reduzido (com oito itens, retirando-se os itens 7 e 10, caping focado na emoxãa ficando este fator com três itens e $\alpha=0,82$ ). Os resultados dessas análises podem ser observados na Tabela 2.

Tabela 2. Índices de ajuste dos modelos da ECOM

\begin{tabular}{lrrllllllll}
\hline Modelos & $\chi^{2}$ & gl & $\Delta \chi^{2}$ & $\Delta$ g.l. & p & GFI & CFI & RMSEA (IC 90\%) & CAIC & ECVI \\
\hline M1 & 81,865 & 35 & & & & 0,94 & 0,93 & $0,072(0,052 ; 0,093)$ & 212,847 & 0,476 \\
M2 & 64,382 & 34 & 17,483 & 1 & 0,00001 & 0,95 & 0,96 & $0,059(0,036 ; 0,081)$ & 201,912 & 0,416 \\
M3 & 52,469 & 32 & 11,913 & 2 & 0,01 & 0,96 & 0,97 & $0,050(0,023 ; 0,074)$ & 203,097 & 0,385 \\
M4 & 21,653 & 17 & 30,816 & 15 & 0,01 & 0,98 & 0,99 & $0,033(0,000 ; 0,069)$ & 146,047 & 0,233 \\
\hline
\end{tabular}

Notas: M1 = Modelo unifatorial; $\mathrm{M} 2$ = Modelo bifatorial; $\mathrm{M} 3$ = Modelo original (trifatorial); $\mathrm{M} 4=$ Modelo original reduzido.

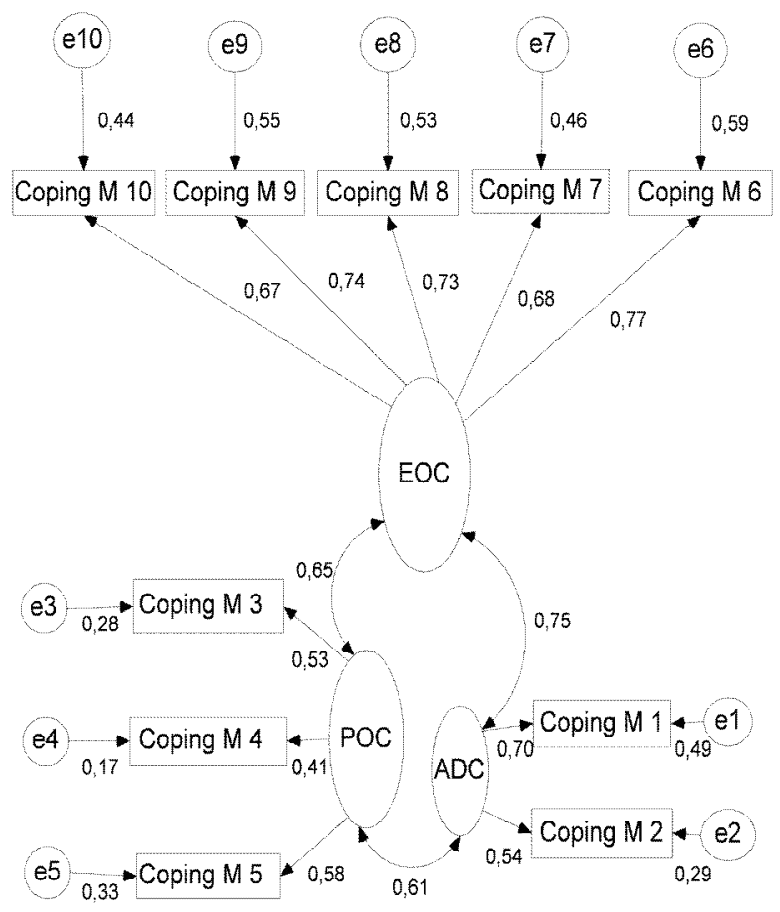

Figura 1. Análise fatorial confirmatória da ECOM

Inicialmente verificou-se o quiquadrado dos modelos divididos por seus graus de liberdade ( $\chi^{2} /$ g.l.), que foram 2,33; 1,89; 1,63 e 1,27, respectivamente.
Este e os demais índices de ajuste mostraram que 0 modelo original (M3) da ECOM se adequou satisfatoriamente aos dados, com melhor ajuste do que 
os modelos unifatorial e bifatorial. Porém, derivou-se deste um modelo tri-fatorial reduzido, formado por 8 itens, o qual apresentou os melhores índices de ajuste e menores valores de CAIC e ECVI. Em suma, estes modelos (MB e M4) se ajustaram satisfatoriamente aos dados e podem ser considerados equivalentes. Todos os pesos de regressão do conjunto de itens foram estatisticamente significativos $(\mathrm{p}<0,001)$. A estrutura original da ECOM com seus pesos fatoriais padronizados pode ser visualizada na Figura 1.

\section{ECOM, ĝ̂neroeidade}

Foi realizada uma MANCOVA com o objetivo de se verificarem os efeitos do gênero nos fatores da ECOM, controlando-se a idade. Verificou-se que as mulheres $(\mathrm{M}=4,00, \mathrm{DP}=0,77)$ pontuaram mais alto do que os homens $(\mathrm{M}=3,42, \mathrm{DP}=1,01)$ no fator copingfocado na emoção e no fator evitação: (mulheres: $\mathrm{M}=3,08, \mathrm{DP}=0,92$ e homens: $\mathrm{M}=2,64, \mathrm{DP}=0,94$ ). Os homens, por outro lado, pontuaram mais alto $(\mathrm{M}=3,13, \mathrm{DP}=0,97)$ do que as mulheres $(\mathrm{M}=3,05$, $\mathrm{DP}=0,91)$ no fator copingfocado no problema.

De acordo com esta análise verificaram-se efeitos multivariados do gênero $(F[3,251]=14,027, p<0,001$, $\lambda$ de Wilks $=0,856, \eta^{2}=0,14$ ) e efeitos univariados especificamente nos fatores coping focado na emoção $\left(F[1,251]=26,993, p<0,001, \eta^{2}=0,10\right)$ e evitação $\left(F[1,251]=14,248, p<0,001, \eta^{2}=0,05\right)$. Mas não se verificou qualquer efeito da idade. Todos os fatores se mostraram positivamente correlacionados, estando 0 fator copingfocado na emoção $(\mathrm{M}=3,71, \mathrm{DP}=0,94)$ mais correlacionado com o fator evitação $(\mathrm{M}=2,86$, $\mathrm{DP}=0,95, \mathrm{r}=0,51, \mathrm{p}<0,001)$ do que 0 fator coping focado no problema $(\mathrm{M}=3,09, \mathrm{DP}=0,94, \mathrm{r}=0,43, \mathrm{p}$ $<0,001)$. O fator copingfocado no problema mostrouse ainda correlacionado com o fator evitação $(r=0,32$, $\mathrm{p}<0,001)$. Com base na inspeção dessas médias, verifica-se que o coping mais usado é o focado na emoção e 0 menos usado é o tipo evitação. Esses resultados se repetem quando se considera a pontuação total de apenas três itens para o coping focado na emoção.

\section{Discussão}

Verificaram-se evidências favoráveis de validade de construto para o modelo original da ECOM baseado na concepção multidimensional de coping Este estudo encontrou resultados semelhantes ao estudo original (Miranda \& Claes, 2009). Especificamente no que diz respeito à estrutura fatorial formada por coping focado na emoção, copingfocado no problema e coping tipo evitação. O percentual de variância encontrado no presente estudo foi apenas sutilmente inferior ao encontrado pelos autores do original, mas igualmente alto; respondendo por mais da metade da variância do construto. Outro dado importante de assinalar é que no presente estudo também foi encontrado um valor próprio "limítrofe", do terceiro fator formado por apenas dois itens. A validade desta estrutura fatorial formada por três fatores, como verificados na literatura especializada (Antoniazzi \& cols., 1998; Carver \& Connor-Smith, 2010; Lazarus \& Folkman, 1984), foi corroborada mediante a abordagem fatorial confirmatória (Browne \& Cudeck, 1993; Byrne, 2001, 2010; Hu \& Bentler, 1999; Tabachnick \& Fidell, 2007), testando-se modelos alternativos como tem sido recomendado na literatura especializada (DiStefano \& Hess, 2005; Thompson, 1995; Weston \& Gore, 2006) com uma amostra de participantes de tamanho adequado para esta análise (Weston \& G ore, 2006).

Considerando a utilidade de medidas breves (Furnham, 2003; Gosling, Rentfrow \& Swann, Jr., 2003), um modelo reduzido, com base no modelo original apresentou índices de ajuste mais satisfatórios, que pode ser considerado equivalente $(\mathrm{McD}$ onald \& Ro, 2002) ao modelo original da ECO M. O ajuste geral do modelo contradisse a regra de valor próprio maior do que 1, como anteriormente verificado (Fabrigar, Wegener, MacCallum \& Strahan, 1999) e também a PA. A decisão de se reterem três fatores na análise fatorial está consoante com a recomendação destes autores de se levar em consideração tanto a teoria como as pesquisas prévias. Esta decisão se demonstrou acertada quando se procedeu ao teste dos modelos. Em suma, a análise fatorial confirmatória foi útil para apoiar a estrutura fatorial teórica de copingpela música representada pela ECOM, a qual se adequou satisfatoriamente aos dados (Laros, 2005).

No que diz respeito à precisão da escala, foi obtido um bom coeficiente de precisão (Anastasi \& Urbina, 2000; Nunnally, 1970, Pasquali, 2003), por meio do alfa de Cronbach, para o fator çpingfocado na emoção. No entanto, índices menores foram encontrados para os outros dois fatores de coping focado no problema e evitação. Esses resultados são fáceis de se entender, uma vez que o coeficiente alfa de Cronbach aumenta na medida em que se aumenta 0 número de itens da escala (Oviedo \& Campo-Árias, 2005), como se verificou no presente estudo. O fator de coping focado no problema conta com apenas três itens e o fator evitação com apenas dois. No contexto nacional, coeficientes iguais a 0,60 , pela Resolução CPF n⿳0 002/2003, têm sido considerados suficientes (Conselho Federal de Psicologia, 2010). Deve-se destacar, ainda, que alfas abaixo do ponto de corte comumente estabelecido de 0,70, porém coeficientes de 0,60 ou menores, têm sido aceitos para medidas 
com fins de pesquisa (Clark \& Watson, 1995; Hair \& cols., 2009; Mueller, 1986).

Uma vez que 0 lambda 2 de Guttman tem sido recomendado para escalas com poucos itens (Tellegen \& Laros, 2004), verificaram-se também esses índices. Tais coeficientes estão muito próximos dos valores de alfa encontrados. Por fim, verificou-se que as correlações médias entre os itens indicam boa consistência interna para os três fatores (Clark \& Watson, 1995).

Tendo em conta o único estudo prévio dessa medida (Miranda \& Claes, 2009), verificaram-se índices de precisão alfa de Cronbach menores nos fatores de coping focado no problema e do tipo evitação, mas similar ao encontrado para o copingfocado na emoção. Os autores justificam teoricamente que esse tipo de coping ou seja, coping através de ouvir música, é predominantemente focado na emoção. Esse dado foi corroborado no presente estudo pela pontuação total dos participantes nesse fator. Por outro lado, também foi confirmado, na amostra total, que o coping de evitação é 0 menos usado. Corroborou-se ainda que esta é uma estratégia de coping mais utilizada pelas mulheres, ao lado do tipo evitação.

Nesse sentido, diferentemente do encontrado no estudo dos autores da escala no contexto canadense, que verificaram que as adolescentes do sexo feminino pontuaram estatisticamente mais alto do que seus pares do sexo masculino em todos os três fatores de coping verificou-se, no presente estudo, que os homens obtiveram mais altos escores no aping focado no problema. No entanto, as diferenças foram estatisticamente significativas nos fatores de coping focado na emoção e evitação e não no copingfocado no problema. Decidiu-se controlar a idade nesta análise, tendo em conta a sua importância para o coping(Carver \& Connor-Smith, 2010; Lazarus \& Folkman, 1984) e uma vez que, no presente estudo, foi considerada uma faixa etária maior do que no estudo original, mas não se verificou qualquer efeito da idade.

Por fim, constatou-se que o coping focado na emoção se correlacionou com o tipo evitação. Esse dado pode parecer consoante com o que destacou Lazarus e Folkman (1984): que o tipo evitação é um tipo de coping focado na emoção. No entanto, esta pesquisa verificou-se que, embora relacionados, esses fatores são distintos. A testagem de modelos baseada na CFA demonstrou que um modelo bifatorial formado pelos fatores coping focado na emoção (incluindo-se 0 tipo cvitação) e 0 fator de copingfocado no problema não apresentou um melhor ajuste do que 0 modelo trifatorial, original da escala.

\section{Considerações finais}

Como toda pesquisa científica, nesta também podem ser verificados aspectos que a limitam. Um primeiro ponto a se abordar diz respeito à natureza da amostra. Mesmo que a escala tenha sido delineada para adolescentes e na presente pesquisa tenha sido considerada uma faixa etária mais ampla, seria interessante testá-la com outras faixas etárias também, como adultos. Pesquisas para testar a estrutura fatorial e consistência interna da ECO M em outras regiões do Brasil são igualmente desejáveis. Por fim, poderia ser importante para a teoria de coping(Lazarus \& Folkman, 1984) verificar a dinâmica, as mudanças no coping pela música em delineamentos longitudinais, observando-se ainda, a importância relativa que grupos etários distintos conferem às estratégias de copingatravés de se ouvir música.

De acordo com os dados desta pesquisa concluise pela validade de construto da ECOM. Novas pesquisas para verificar a consistência dessa estrutura fatorial são todavia necessárias. Além disso, mesmo tendo sido encontrados coeficientes de precisão menores do que no estudo original, pondera-se que esta medida pode ser utilizada com fins de pesquisa. Ademais, as correlações médias entre os itens indicaram sua consistência interna. Por fim, é importante que novos estudos sejam realizados, apontando correlações da ECO M com outras variáveis, como o bem-estar subjetivo e traços de personalidade. Pode-se verificar, por exemplo, a eficácia das estratégias de caping por ouvir música na redução do estresse.

\section{Referências}

Anastasi, A., \& Urbina, S. (2000). Tetagm psicoógica. Porto Alegre: ArtMed.

Antoniazzi, A. S., Dell'aglio, D. D., \& Bandeira, D. R. (1998). O conceito de coping uma revisão teórica. EstudosdePsicloga (Natal), 3(2), 273-294.

Antoniazzi, A. S., Souza, L. K., \& Hutz, C. S. (2009). Copingem situações específicas, bem-estar subjetivo e autoestima em adolescentes. Gerais Revista Inteinstiturional dePsiclogia, 2(1), 34-42.

Aspinwall, L. G., \& Taylor, S. E. (1992). Modeling cognitive adaptation: a longitudinal investigation of the impact of individual differences and coping on college adjustment and performance. Jamal of Pessanalityand Social Psychdogy, 63(6), 989-1003.

Balbinotti, M. A. A., Barbosa, M. L. L., Wiethaeuper, D., \& Teodoro, M. L. M. (2006). Estrutura fatorial 
do Inventário Multifatorial de Coping para adolescentes (IMCA-43). PsicoPUCRS, 37(2), 123130.

Barker, D. B. (2007). Antecedents of stressful experiences: depressive symptoms, self-esteem, gender and coping. Intemational Jaumal of Stress Managemet, 14(4), 339-349.

Boer, D. (2009). Musicmakes thepeeplecometogther: soial functions of misic listering for young peeple across altures (Tese de Doutorado). Victoria University of Wellington, New Zealand.

Browne, M. W., \& Cudeck, R. (1993). Alternative ways of assessing model fit. Em K. A. Bollen \& J. S. Long (Eds.), Tetingstructural equation modds (pp. 136162). Newbury Park, CA: Sage.

Bueno, V. F, \& Macedo, E. C. (2004). Julgamento de estados emocionais em faces esquemáticas por meio da música por crianças. Psicdogja: Teøia e Prática, $6(2), 27-36$.

Bueno, V. F., \& Bergamasco, N. H. P. (2008). Efeito da associação de sabor e música sobre o estado de ânimo de crianças. Estudos de Psiølogia (Campinas), 25(3), 385-393.

Byrne, B. M. (2001). Structural equation modding with AMOS: basic concepts applications and programming Mahwah: Lawrence Erlbaum Associates.

Byrne, B. M. (2010). Structural equation modding with AMOS: basic concepts applications and progamming Nova iorque, USA: Routledge, Taylor and Francis Group.

Carver, C. S., \& Connor-Smith, J. (2010). Personality and coping. Annual Review of Psydhogy, 61(1), 679704.

Cattell, R. B., \& Saunders D. R. (1954). Musical preferences and personality diagnosis: a factorization of one hundred and twenty themes. Jaumal of Social Psydhdogy, 39(1), 3-24.

Clark, L. A., \& Watson, D. (1995). Constructing validity: basic issues in objective scale development. Psychdogical Assesment, 7(3), 309-319.

Dela Coleta, A. S. M., \& Dela Coleta, M. F. (2008). Fatores de estresse ocupacional e coping entre policiais civis. PsicoUSF, 13(1), 59-68.

Diniz, S. S., \& Zanini, D. S. (2010). Relação entre fatores de personalidade e estratégias de coping em adolescentes. PsicoUSF, 15(1), 71-80.
DiStefano, C., \& Hess, B. (2005). Using confirmatory factor analysis for construct validation. Joumal of Psychoeducational Assssment, 23(3), 225-241.

Fabrigar, L. R., Wegener, D. T., MacCallum, R. C., \& Strahan, E. J. (1999). Evaluating the use of exploratory factor analysis in psychological research. Psychdogical Methods, 4(3), 272-299.

Folkman, S., Lazarus, R. S., Dunkel-Schetter, C., DeLongis, A. \& Gruen, R. J. (1986). Dynamics of a stressful encounter: cognitive appraisal, coping, and encounter outcomes. Jaumal of Pesonality and Social Psychdogy, 50(5), 992-1003.

Furnham, A. (2003). Belief in a just world: research progress over the past decade. Pescnality and Individual Differences, 34(5), 795-817.

Garson, G. D. (2010). Structural equation modeling. Statnotes Topics in MultivariateA Aalysis Recuperado: 02 de outubro de 2010. Disponível: http:/ / faculty.chass.ncsu.edu/ garson/ PA 765/ struct ur.htm

Gosling, S. D., Rentfrow, P. J., \& Swann, W. B., Jr. (2003). A very brief measure of the Big Five Personality domains. Jamal of Reseren in Pessonality, 37(6), 504-528.

Hair, J. F., Black, W. C., Babin, B. J., Anderson, R. E., \& Tatham, R. L. (2009). Análisemiltivariada dedados Porto Alegre, RS: ArtMed.

Hayton, J. C., Allen, D. G., \& Scarpello, V. (2004). Factor retention decisions in exploratory factor analysis. Organizational Reserch Methods 7(2), 191205.

Hetland, L. (2000). Listening to music enhances spatialtemporal reasoning: evidence for the "Mozart effect." Jaumal of Aesthtic Education, 34(3-4), 105148.

Hu, L. T., \& Bentler, P. M. (1995). Evaluating model fit. Em R. H. Hoyle (O rg.). Structural equation modding conepts, issues, and applications (pp. 76-99). Thousand O aks, CA: Sage.

Hu, L. T., \& Bentler, P. M. (1999). Cutoff criteria for fit indexes in covariance structure analysis: conventional criteria versus new alternatives. Structural Equation Modding 6(1), 1-55.

Laros, J. A. (2005). O uso da análise fatorial: algumas diretrizes para pesquisadores. Em L. Pasquali (O rg.), Análise fadtoial para pesquisadares (pp. 163184). Brasília: LabPAM. 
Lazarus, R. S., \& Folkman, S. (1984). Stress, appraisal, and coping Nova Iorque: Springer.

Lisboa, C., Koller, S. H., Ribas, F. F., Bitencourt, K., Oliveira, L., Porciuncula, L. P., \& De Marchi, R. B. (2002). Estratégias de coping de crianças vítimas e não vítimas de violência doméstica. Psiđagja: Rellexão\& Cútica, 11(2), 395-411.

MacCallum, R. C., \& Austin, J. T. (2000). Applications of structural equation modeling in psychological research. Annual Reviewof Psychdogy, 51(1), 201-226.

McD onald, R. P., \& Ho, M. H. R. (2002). Principles and practice in reporting structural equation analyses. Psychdogical Methoos, 7(1), 64-82.

Miranda, D., \& Claes, M. (2009). Music listening, coping, peer affiliation and depression in adolescence. Psychdogy of Music 37(2), 215-233.

Mitchell, L. A., MacD onald, R. A. R., Knussen, C., \& Serpell, M. G. (2007). A survey investigation of the effects of music listening on chronic pain. Psychdogy of Music, 35(1), 39-59.

Mueller, D. J. (1986). Measuingsoial attitudes a handbook for rearchers and practitioness Nova Iorque: Teachers College Press.

North, A. C., Hargreaves, D. J., \& O'Neill, S. A. (2000). The importance of music to adolescents. BritishJaumal of Educational Psydhogy, 70(2), 255-272.

Nunnally, J. C. (1970). Introduction to psydhdogical masurement. Tokio: McG raw-Hill.

Oviedo, H. C., \& Campo-Árias, A. (2005). Aproximación al uso del coeficiente alfa de Cronbach. Reista Cdombiama de Psiquiatría, 34 (4), 527-580.

Panzini, R. G., \& Bandeira, D. R. (2007). Coping (enfrentamento) religioso/ espiritual. Rexista de Psiquiatria Clínica, 34, suplemento 1, 126-135.

Pasquali, L. (2003). Psicomenia: teeria dos testes na psicdoga ena eurcaçãa Petrópolis: Vozes.

Pimentel, C. E., \& Donnelly, E. D. O. P. (2008). A relação da preferência musical com os cinco grandes fatores da personalidade. Psicogia: Ciêna \& Profissãa, 28(4), 682-713.

Premuzic-Chamorro, T., \& Furnham, A. (2007). Personality and music: can traits explain how people use music in everyday life? BritishJaumal of Psydhogy, 98(2), 175-185.
Raimundo, R. C. P., \& Pinto, M. A. P. M. (2006). Stress e estratégias de copingem crianças e adolescentes em contexto escolar. Alehia, 24, 09-19.

Rentfrow, P. J., \& Gosling, S. D (2003). The do re mi's of everyday life: the structure and personality correlates of music preference. Jaumal of Personality and Social Psychdogy, 84(6), 1226-1236.

Santos, K., \& Martendal, L. (2008). Coping e adoecimento cardíaco em um trabalhador da saúde. Psicdoja Argumeto, 26(55), 281-292

Schellenberg, E. G., \& Hallam, S. (2005). Music listening and cognitive abilities in 10 and 11 year olds: The Blur effect. Annals of the New York Acadkmy of Saiences, 1060(1), 202-209.

Schwartz, K. D., \& Fouts, G. T. (2003). Music preferences, personality style, and developmental issues of adolescents. Jaumal of YauthandAddesence, 32(3), 205-221.

Tabachnick, B. G., \& Fidell, L. S. (2007). Using multivariate statistics. Needham Heights, MA: Allyn \& Bacon.

Tamayo, M. R., \& Tróccoli, B. T. (2002). Exaustão emocional: relações com a percepção de suporte organizacional com as estratégias de coping no trabalho. EstudosdePsicdoja, 7(1), 37-46.

Tellegen, P. J., \& Laros, J. A. (2004). Cultural bias in the SONR test: comparative study of Brazilian and Dutch children. Psicloga: Teria e Pesquisa, 20(2), 103-111.

Terry, D. J. (1991). Coping resources and situational appraisals as predictors of coping behavior. Pessanality and Indvidual Differenes, 12(10), 10311047.

Thompson, B. (2005). Explaratary and confimatary factor analysis Undastanding concepts and applications Washington: American Psychological Association.

Vera-Noriega, J. A., Albuquerque, J. F. B., Laborín, J. A., \& Pimentel, C. E. (2003). Coping em uma população do Nordeste brasileiro. Avaliação Psicdógica, 1(3), 17-27.

Weston, R., \& Gore, P. A. (2006). A brief guide to structural equation modeling. The Cansding Psychdogist, 34(5), 719-751.

Zwick, R., \& Velicer, W. F. (1986). Comparison of five rules for determining the number of components to retain. Psychdogial Bullein, 99(3), 432-442. 
Reedidoem25/ 10/ 2010

Reformuladoem15/ 02/ 2012

Aprovadoem23/ 02/ 2012

Os autores agradecem ao CNPq pela Bolsa de Doutorado e Iniciação Científica concedidas ao primeiro e quinto autor respectivamente. Agradecem também a D ave Miranda por ter disponibilizado a CopingbyMusicListeningScalee feito comentários valiosos acerca desta pesquisa.

Sobre os autores:

Carlos E duardo Pimentel é doutorando no Programa de Pós-Graduação em Psicologia Social, do Trabalho e das Organizações na Universidade de Brasília, mestre em Psicologia Social, formado em Psicologia e licenciado em Psicologia pela Universidade Federal da Paraíba. É bolsista de Doutorado do CNPq. Email: carlosepimentel@bol.com.br

Marlizete Maldonado Vargas é doutora em Psicologia, Ciência e Profissão pela Pontifícia Universidade Católica de Campinas, professora adjunta III do curso de graduação em Psicologia e do Mestrado em Saúde e Ambiente da Universidade Tiradentes. Email: marlizete@uol.com.br

Thiago Oliveira de Almeida é formado em psicologia pela Universidade Tiradentes. Atualmente faz PósGraduação (especialização) em Psicossomática pelo Instituto Junguiano da Bahia e MBA em Administração com ênfase em RH pela Fundação Getúlio Vargas. Email: t.oliveira97@ yahoo.com.br

Viviane Andrade Prado Maynart é formada em psicologia pela Universidade Tiradentes. Atualmente faz PósGraduação (especialização) em Psicologia do Trânsito na Faculdade Pio Décimo. E-mail: vivianemaynart@ig.com.br

Sarah Monteiro de C. T. Figueiredo é estudante do curso de Formação em Psicologia na Universidade Tiradentes e bolsista de Iniciação Científica do CNPq. Email: sarinha_bis@ hotmail.com

Contato com os autores:

Universidade de Brasília, Instituto de Psicologia (IP). Programa de Pós-Graduação em Psicologia Social, do Trabalho e das Organizações. Campus Universitário Darcy Ribeiro. Asa Norte. 70919-970, Brasília, DF. CaixaPostal: 4500.

Email: carlospimentel@unb.br 